\title{
Utilidad del Inventario de Depresión Rasgo-Estado (IDERE) para el diagnóstico clínico. Un estudio con muestras de cuatro países iberoamericanos
}

\author{
Marta Martín-Carbonell1*, Antonio Riquelme-Marín², Juan Manuel Ortigosa-Quiles ${ }^{2}$, Rosa Martha Meda ${ }^{3}$, \\ Ara Mercedes Cerquera-Córdoba ${ }^{4}$, José A. Enríquez-Santos ${ }^{1}$ y Raquel Pérez-Díaz ${ }^{5}$ \\ ${ }^{1}$ Universidad de la Habana (Cuba) \\ ${ }^{2}$ Universidad de Murcia (España) \\ ${ }^{3}$ Universidad de Guadalajara (México) \\ ${ }^{4}$ Universidad Pontificia Bolivariana (Colombia) \\ ${ }^{5}$ Centro de Investigaciones sobre Longevidad, Envejecimiento y Salud (CITED) (Cuba).
}

\begin{abstract}
Resumen: El Inventario de Depresión Rasgo-Estado (IDERE) fue diseñado para evaluar la depresión como estado y como rasgo. Este trabajo se propuso estudiar su utilidad para identificar casos en los que se estima depresión clínica de acuerdo a los criterios del DSM-IV. La muestra estuvo constituida por 1984 sujetos de cuatro países: 503 españoles, 330 cubanos, 477 colombianos y 674 mexicanos. Se utilizó la Escala del Centro de Estudios Epidemiológicos de la Depresión (CES-D) para estudiar la validez concurrente y el Inventario de Síntomas Depresivos de Riquelme para identificar casos que cumplieran criterios del DSM-IV para diagnóstico de un episodio depresivo. El análisis de las curvas ROC por grupos edades y géneros, así como para Cuba, México y España, mostró que ambas subescalas tenían buena precisión, sin embargo para la muestra colombiana ninguna de las subescalas obtuvo resultados aceptables. Se encontraron diferencias significativas en puntuaciones del IDERE atribuibles a sexo, país, edad; y a la interacción entre estas variables. Ambas subescalas obtuvieron correlaciones significativas y positivas con el CES-D. Los resultados hablan a favor de la validez de criterio y convergente del IDERE y de su valor para la identificación de personas con posible depresión en países iberoamericanos.
\end{abstract}

Palabras clave: Depresión; psicodiagnóstico; estudio transcultural; IDERE.

\section{Introducción}

La depresión es un estado con una elevada prevalencia en la población, con una baja tasa de detección en el ámbito de los sistemas de salud y con un importante impacto en la calidad de vida de las personas, que afecta además otros ámbitos como el familiar, físico, social, laboral y económico (Riquelme, Martín, Ortigosa y Lechuga, 2008). Estas son algunas de las razones por las cuales el desarrollo de instrumentos para la detección de la sintomatología depresiva ha sido un área de investigación muy activa y con un creciente desarrollo en el ámbito hispanoamericano (González, Martín, Grau y Lorenzo, 2007). Sin embargo, a las escalas para la evaluación de la depresión se les señala que tienen una escasa precisión conceptual, ausencia de normas de aplicabilidad y propiedades psicométricas insuficientes (Spielberger, Ritterband, Reheiser y Brunner, 2003; Dowd, 2004; González et al., 2007; Cabañero-Martínez, Cabrero-García, RichartMartínez, Muñoz-Mendoza y Reig-Ferrer, 2007) por lo que resultan necesarios los estudios dirigidos a precisar el valor

* Dirección para correspondencia [Correspondence address] Marta Martín Carbonell. Domicilio: Calle Infanta 1506, apartamento 14G. entre Estévez y Santa Rosa. 10600, Cerro. La Habana (Cuba). E-mail: mmartin@infomed.sld.cu
Title: Usefulness of the State-Trait Depression Inventory (IDERE) for clinical diagnosis. A study containing samples from four Iberian-American nations.

Abstract: State-Trait Depression Inventory (IDERE) is a self-evaluating inventory designed to evaluate depression as a state and as a trait. This work set a goal to study its usefulness to identify cases in which clinical depression -based on the DSM-IV criteria- are estimated. The sample was made up of 1984 individuals from four countries: 503 Spaniards, $330 \mathrm{Cu}$ bans, 477 Colombians and 674 Mexicans. The Scale of the Center for Epidemiological Studies on Depression (CES-D) was used in an effort to assess the concurrent validity and the Riquelme Inventory of Depressive Symptoms to pinpoint cases that comply with the DSM-VI criteria for the diagnosis of a depressive episode. The analysis of the ROC curves by age and gender groups, as well as for Cuba, Mexico and Spain, showed good accuracy rate in both scales. However, for the Colombian sample none of the subscales yielded acceptable outcomes. Significant differences were found in terms of gender, country, age and the interaction among these variables. Both subscales produced significant and positive correlations with CES-D. The results speak in favor of the validity of criteria and convergence with IDERE, as well as its value for the identification of people suffering from possible depression in Hispanic American countries.

Key words: Depression; psychodiagnosis; IDERE; transcultural studies.

diagnóstico de los instrumentos que actualmente se usan más en la práctica clínica.

Uno de los problemas que afecta a la precisión de los instrumentos está relacionado con las dificultades conceptuales en torno al término depresión. La idea vigente sobre la depresión en los manuales de diagnóstico supone la visión de un trastorno periódico y limitado en el tiempo. Sin embargo, diferentes autores consideran que sería más fructífero plantear un modelo dimensional que considerara la relación de lo psicopatológico con los procesos normales, más que el modelo categorial vigente en la actualidad (Agudelo, Spielberger y Buela Casal, 2007; Mellor y Aragona, 2009; Díaz, Blanco, Horcajo y Valle, 2007). Las críticas al sistema categorial se encuentran desde el mismo DSM-IV-TR (American Psychiatric Association, 2000) en el que se indica por ejemplo, que no debe asumirse que las categorías clínicas sean entidades discretas con límites absolutos.

Diversas investigaciones ponen de manifiesto la posibilidad de considerar la depresión a lo largo de un continuo, donde en el extremo menos grave se encontrarían estados reactivos transitorios a situaciones concretas, pasando por la personalidad depresiva; y en el extremo más grave el trastorno depresivo mayor (Grau, Martín y Portero, 1993; Spielberger et al, 2003).

Partiendo de esta concepción dimensional, en 1986 se comenzó a trabajar en Cuba en el desarrollo del Inventario 
de Depresión Rasgo-Estado (IDERE), con la pretensión de contar con un instrumento que permitiera por una parte, identificar a las personas vulnerables a presentar estados depresivos, o sea, que tienen una alta depresión como rasgo o depresión personal; así como identificar a las personas que están sufriendo un estado depresivo que provoque malestar emocional significativo, aunque este no necesariamente se manifieste como una depresión que cumpla todos los criterios diagnósticos establecidos en los manuales de psiquiatría (Ramírez, Grau, Martín y Grau, 1986; Martín, Grau, Ramírez y Grau, 2001; Martín, Grau y Grau, 2003).

Existe también el Cuestionario de Depresión EstadoRasgo (ST-DEP) desarrollado de manera totalmente independiente por otros autores (Spielberger, Carretero-Dios, De Los Santos-Roig, Buela-Casal, 2002a y 2002b; Spielberger, Carretero-Dios, De los Santos-Roig, Buela-Casal, 2004; Agudelo, Carretero-Dios, Blanco, Pitti, Spielberger y BuelaCasal, 2005; Ocampo, 2007; Agudelo, 2009), sin embargo, a pesar de que ambos instrumentos se basan en un enfoque dimensional de la depresión, la finalidad del ST-DEP es constituir una medida específica del componente afectivo de la depresión rasgo-estado a partir de los modelos de afectividad positiva vs. negativa, mientras que el IDERE se centra más en los componentes cognitivo-valorativos.

Así, se elaboró un inventario autoevaluativo que consta de dos subescalas que se pueden aplicar de manera conjunta o independiente. En ambas subescalas se incluyeron ítems directos e inversos de depresión con el objetivo de detectar niveles bajos de malestar depresivo que pueden tener un impacto similar, o superior incluso que la depresión mayor, tanto en el funcionamiento físico como en la discapacidad cotidiana, como ocurre con la llamada "depresión subclínica" o "depresión menor" (Beekman, Deeg, van Tilburg, Smit, Hooijer y van Tilburg, 1995; Hybels, Blazer y Piper, 2001)

Los estudios psicométricos iniciales encontraron una buena consistencia interna para ambas subescalas del IDERE (correlaciones item-item e ítem-escala con niveles de significación entre .05 y .01 para todas las asociaciones, y coeficiente alfa de Cronbach de .78 para la escala de depresión rasgo y de .79 para la escala de depresión como estado). Se ha investigado la estabilidad de las puntuaciones de la subescala rasgo a los quince días de la primera aplicación, encontrándose correlaciones de .95 para los niveles moderados y bajos; y de .89 para la depresión alta (Ramírez et al., 1986). Un estudio con 105 adolescentes chilenos que recibieron un taller psicoeducativo de tres meses de duración que tenía como objetivo favorece la integración de los distintos estados emocionales y lograr la expresión de los afectos de una manera más saludable, no encontró diferencias significativas en los niveles de depresión estado-rasgo en los 61 estudiantes que al inicio del taller tenían buena salud emocional, encontrándose correlaciones significativas entre las puntuaciones obtenidas en las dos aplicaciones (entre .35 y .41), mientras que sí detectó cambios significativos en ambas subescalas en los 44 alumnos que tenían problemas emocionales, lo que habla a favor de la sensibilidad del IDERE para detectar efectos de las intervenciones psicológicas (Lechuga, 2007) .

Con respecto a su validez externa, se ha encontrado (Ramírez et al., 1986) en población normal que es capaz de discriminar con éxito a personas con altos niveles de depresión rasgo de acuerdo al criterio de sus compañeros y a su propia autoevaluación (92.3\% para la escala estado y $96.9 \%$ la escala rasgo), así como identificar adecuadamente a pacientes con enfermedades cardiovasculares que presentan problemas de depresión que requieren atención psicológica (Martín, 2003).

Se ha encontrado que muestra correlaciones significativas y positivas con el IDARE en 302 adultos cubanos con enfermedades cardiovasculares (entre $\mathrm{r}=.558 \mathrm{y} \mathrm{r}=.701$ ), y en un estudio con 3251 adolescentes chilenos (entre $r=.647$ y $r=$.794). También se ha estudiado en la muestra de enfermos cardiovasculares las correlaciones del IDERE con diversas subescalas del PSICRON, que es un inventario multidimensional para la evaluación psicológica de personas con enfermedades crónicas. En este estudio se encontraron correlaciones significativas y positivas con la subescalas de depresión (IDERE-E r= .462 e IDERE-R $\mathrm{r}=.513$ ) y pesimismo (IDERE-E $r=.565$ e IDERE-R $r=.594)$; y ninguna correlación con la escala optimismo (IDERE-E r=.067 e IDERE-R r=.024) (Martín, 2003).

Actualmente el IDERE es utilizado en la mayor parte de los centros de salud de Cuba y también en diferentes instituciones de España, México, Chile, Perú y Colombia. Se ha usado también en diversas investigaciones: para el diagnóstico de la depresión en enfermos crónicos (Martín y Grau, 1991), para evaluar la calidad de vida de pacientes oncológicos terminales (Jiménez, 1993), los problemas de burnout en los servicios de cuidados paliativos (Chacón, Grau, Martín, 1994), para la selección de pacientes con dolor crónico candidatos a implantes de sistemas (Navarro, Marchena, González, Manjón, Pallarés y Martín, 1996),con dolor agudo(Martín y Lechuga, 2003) y en ancianos con dolor crónico (Martín y Lechuga, 2004), entre otros; se han presentado trabajos con el IDERE en varios eventos nacionales e internacionales (Martín y Grau, 1991; Grau et. al., 1991b; Martín y Grau, 1993b; ) y aparece en varios manuales de instrumentos de evaluación psicológica (González, Martín, Grau y Lorenzo, 2007; Martín, Grau, Ramírez y Grau, 2005).

Este trabajo se propone valorar la utilidad del IDERE para identificar a personas con un trastorno depresivo que responda a los criterios diagnósticos establecidos, pues aunque el propósito original del IDERE ya se comentó que no era este, su difusión hace necesario que se puntualice su alcance. Por esta razón, se estudió su sensibilidad y especificidad para identificar casos en los que se estima depresión de acuerdo a los criterios del DSM-IV. También se investigó su validez concurrente mediante el estudio de su correlación con el Cuestionario de Depresión de Radolff (Radolff, 1977) que es un instrumento utilizado para el pesquisaje de la depresión validado en los cuatro países que participaron en esta investigación (Enríquez, Martín, Riquelme, 2008; Campos, 
Díaz, Rueda, Cadena y Hernández, 2007; Zunzunegui, Delgado, Pérez, Yagüe, Illescas. y León, 1998; Soler, Pérez-Sola, Puigdemont, Pérez-Blanco, Figueres y Álvarez, 1994; Guarnaccia, Angel y Worobey, 1989).

\section{Método}

\section{Participantes}

La muestra no probabilística estuvo constituida por 1984 sujetos de cuatro países: 503 españoles, 330 cubanos, 477 colombianos y 674 mexicanos, con edades comprendidas entre los 18 y los 106 años $(M=49.2, D E=22.61)$ predominando el sexo femenino en todos los países (60.6\% para España, $60.9 \%$ para Cuba, $52.6 \%$ para Colombia y $64.5 \%$ para México). La edad promedio para la muestra española fue de 46.7, con una desviación típica (DE) de 21.6; la media de edad para Cuba fue de 52.7 con una $\mathrm{DE}=20.9$; en el caso de Colombia el promedio de edad era 57.19, DE $=20.6$; y para México la media fue de 46.6 con una $\mathrm{DE}=24.10$. Se dividió en tres subgrupos de edades: uno de jóvenes integrado por personas de menos de 30 años en el que entró el 39\% de la muestra española, $18.8 \%$ de la cubana, $17.4 \%$ de la colombiana y $38.7 \%$ de la mexicana; el segundo grupo de edad incluye a los sujetos con más de 35 y menos de 55 años con el $31.4 \%$ de los españoles, $20.3 \%$ de los cubanos, $25.2 \%$ de los colombianos y $23 \%$ de los mexicanos, así como un tercer grupos de ancianos de más de 65 años con el $27.3 \%$ de los españoles, $46.4 \%$ de los cubanos, $49.5 \%$ de los colombianos y $35.3 \%$ de los mexicanos.

Refirieron padecer de alguna enfermedad el $29 \%$ de los españoles, el $62.4 \%$ de los cubanos, $24.9 \%$ de los colombianos y $40.1 \%$ de los mexicanos.

Resultaron analfabetos $4.2 \%$ de los españoles, $1.8 \%$ de los cubanos, $3.6 \%$ de los colombianos y $14.7 \%$ de los mexicanos; tenían escolaridad primaria $31.6 \%$ de los españoles, $10 \%$ de los cubanos, $31.7 \%$ de los colombianos y $21.5 \%$ de los mexicanos; con nivel medio se encontraron al $26 \%$ de los españoles, $57.3 \%$ de los cubanos, $35.6 \%$ de los colombianos y $16 \%$ de los mexicanos, el resto de los casos de los respectivos países refirieron tener nivel superior. Tenían vínculo laboral el $34.2 \%$ de los españoles, $40.3 \%$ de la muestra cubana, $50.9 \%$ de la colombiana y $27.3 \%$ de la mexicana.

\section{Instrumentos}

\section{Inventario de Depresión Rasgo-Estado (IDERE)}

El IDERE es un inventario autoevaluativo diseñado para evaluar dos formas relativamente independientes de la depresión: la depresión como estado (condición emocional transitoria) y la depresión como rasgo (propensión a sufrir estados depresivos como una cualidad de la personalidad relativamente estable).

La escala que evalúa la depresión como estado permite identificar de manera rápida a las personas que tienen sínto- mas depresivos, así como sentimientos de tristeza reactivos a situaciones de pérdida o amenaza, que no necesariamente se estructuran como un trastorno depresivo, aunque provoquen malestar e incapacidad. Tiene 20 ítems cuyas respuestas toman valores de 1 a 4 . Trece de estos ítems por su contenido, son positivos en los estados depresivos, mientras que los restantes son ítems antagónicos con la depresión. El sujeto debe seleccionar la alternativa que mejor describe su estado en ese momento y tiene cuatro opciones de respuestas: No en lo absoluto (que vale 1 punto), Un poco (vale 2 puntos), Bastante (vale 3 puntos) y Mucho (vale 4 puntos).

La escala de depresión como rasgo permite identificar a las personas que tienen propensión a sufrir estados depresivos, y también ofrece información sobre la estabilidad de los síntomas depresivos. Consta de 22 ítems (10 inversos y 12 directos) que también adquieren valores de 1 a 4 puntos y las opciones de respuestas son: Casi nunca (que vale 1 punto), Algunas veces (vale 2 puntos), frecuentemente (3 puntos) y casi siempre (4 puntos).

La calificación se obtiene mediante la suma algebraica de las puntuaciones de los ítems directos e inversos, a cuyo resultado se le suma el valor 50 para conseguir que todos los valores sean positivos. Las puntuaciones oscilan entre $20 \mathrm{y}$ 80 puntos para la escala estado; y entre 22 y 88 puntos para la escala rasgo. Tanto la depresión estado como la depresión rasgo se califican en tres niveles: bajo, medio y alto. De valor clínico son las puntuaciones que se catalogan como altas. Las cifras que están cercanas a los puntos de corte pueden resultar de interés, pero realmente tienen valor diagnóstico los valores superiores a 50. Es recomendable que las personas que obtengan valores altos en el IDERE sean valoradas en consulta médica o psicológica para precisar el diagnóstico.

En esta investigación se realizaron cambios en la redacción de algunos ítems para corregir la doble negación y se agregó un ítem experimental a la escala rasgo. Así en la versión que se investiga en este estudio, en la escala estado hay 13 ítems directos de depresión y 7 inversos; y en la escala rasgo hay 13 ítems directos y 10 inversos.

\section{Escala del Centro de Estudios Epidemiológicos de la Depresión (Center for Epidemiologic Studies-Depression Scale, CES-D)}

La Escala del Centro de Estudios Epidemiológicos de la Depresión (CES-D) es un cuestionario de 20 ítems, con cuatro opciones de respuestas que indican la frecuencia y/o intensidad de la presentación de cada ítem. La mayoría de los estudios coinciden en encontrar 4 factores, aunque su composición varía por lo que hay consenso acerca de que la estructura factorial del CES-D depende de las características de las muestras (Guarnaccia et.al., 1989; Vázquez, Blanco, López, 2007; Helmesa y Nielson, 1998). La consistencia interna del CES-D evaluada mediante el coeficiente alfa de Cronbach es satisfactoria, con valores entre .75 y .89 para la población cubana, mexicana y española (Vázquez et.al, 2007; Salgado y Maldonado, 1994; Campos et. al, 2007; So- 
ler, Pérez-Sola, Puigdemont, Pérez-Blanco, Figueres y Alvarez, 1997; Zunzunegui et. al., 1998).

\section{Inventario de sintomas depresivos de Riquelme}

Fue elaborado y validado en la Universidad de Murcia (Riquelme, Buendía y López, 2006) y se ha obtenido información acerca de sus propiedades psicométricas para la población cubana (Pérez, Riquelme, Martín, Enriquez y Ortigosa, 2009). Comprende un total de 56 síntomas, expresados en 58 ítems en los que se recogen los criterios clínicos de los sistemas DSM-IV-TR y CIE- 10. La valoración de los síntomas, se realiza según su presencia durante las dos últimas semanas, con las opciones de respuesta: Nunca, Casi Nunca, Con frecuencia, la mayor parte del tiempo. En esta investigación se usó para seleccionar los casos con posible depresión clínica.

\section{Procedimiento}

Los datos provienen de un estudio más amplio, que utiliza diferentes instrumentos integrados en un único modelo de entrevista estructurada, que incluye además las preguntas referidas a variables sociodemográficas y de salud, el cual fue aplicado de manera individual por encuestadores especialmente entrenados para esta investigación.

Los casos de Cuba provienen de la Habana y se captaron en asociaciones de ancianos, centros de trabajo y estudio, y residentes del municipio Cerro, así como en la consulta de Psicología del Centro de Investigaciones sobre Longevidad, Envejecimiento y Salud. (CITED). Los casos de México provienen de Ciudad Guzmán y la Zona Metropolita de Guadalajara en Jalisco y de la Ciudad de Morelia en Michoacán, captados en universidades, oficinas de gobierno y asilos y estancias de día. En Colombia se tomaron los datos de la Ciudad de Bucaramanga y su área metropolitana, en centros de atención día y en la Unidad Deportiva el Parque de Floridablanca Santander. Los datos de España fueron tomados de la Región de Murcia a través de la heteroaplicación del modelo de entrevista tanto en población general como en asociaciones sociales, centros de día y residencias de ancianos.

Los participantes fueron reclutados a través de la técnica «bola de nieve », consistente en la ampliación progresiva de la muestra a partir de los contactos proporcionados por los participantes inicialmente seleccionados por los miembros del equipo de investigación siguiendo un criterio de conveniencia.

De acuerdo a los lineamientos internacionales sobre los aspectos éticos de la investigación biomédica con humanos (Consejo de Organizaciones Internacionales de Ciencias Médicas y Organización Mundial de la Salud, 1982) a todos los posibles participantes se les explicó el objetivo de la in- vestigación y se les solicitó el consentimiento para utilizar la información proporcionada con fines de investigación, asegurando la privacidad y confidencialidad. Las personas que como resultado de su participación en el estudio, solicitaron atención psicológica especializada fueron derivadas a profesionales de la salud de su área de atención.

Para el presente trabajo se estimó que podrían presentar depresión clínica las personas que cumplieran el criterio de presentar en las últimas dos semanas con frecuencia o la mayor parte del tiempo al menos 5 de los síntomas (incluyendo el $1 \mathrm{y} /$ o el 2 necesariamente) que señala el DSM-IV para el diagnóstico de un episodio depresivo. Esta información se obtuvo a partir de las respuestas al Inventario de Síntomas, el cual fue analizado para cada caso con este fin por los dos primeros autores de este trabajo.

El 21.8\% de la muestra total cumplió los criterios de depresión clínica estimada: $17.9 \%$ de los españoles, $33 \%$ de los cubanos, $9 \%$ de los colombianos y $28.2 \%$ de los mexicanos.

\section{Análisis de los datos}

Para precisar el valor diagnóstico del IDERE se utilizó la metodología análisis de las curvas Receiver-Operating Characteristic (ROC). Se calculó el área bajo la curva por países, sexos y grupos de edad. Se empleó la estadística descriptiva de la escala para la muestra total, por países, sexos y grupos de edades. Se utilizó el ANOVA multifactorial para identificar las diferencias en los niveles de depresión estado y rasgo atribuibles a la nacionalidad, el género y la edad. Las correlaciones de las subescalas del IDERE con el CES-D se obtuvieron mediante el Coeficiente de correlación de Pearson.

\section{Resultados}

Los resultados de los diferentes análisis de las curvas ROC se resumen en la Tabla 1.

Como puede observarse para Cuba, México y España ambas subescalas del IDERE obtienen resultados significati$\operatorname{vos}(\mathrm{p}=.000)$ que indican una buena precisión, lo que se corrobora cuando se observa que las curvas están cercanas al extremo superior izquierdo (Figuras 1 al 3); sin embargo, para la muestra colombiana ninguna de las subescalas obtiene resultados aceptables. Nótese como en todos los casos, la escala estado obtiene mejores indicadores que la de rasgo. Por grupos de edades y por género se encuentra que ambas subescalas muestran también una buena precisión.

La Tabla 2 permite visualizar los puntos de corte recomendados para España, Cuba y México. Estos puntos de corte se seleccionaron teniendo en cuenta que son los que maximizan la sensibilidad (igual o mayor al $70 \%$ ) perjudicando lo menos posible la especificidad (valor de 1especificidad igual o menor al 30\%). 
Tabla 1. Resumen de resultados de ROC por países, edades y sexos

\begin{tabular}{|c|c|c|c|c|c|}
\hline \multirow[t]{2}{*}{ Variables resultado de contraste } & \multirow[t]{2}{*}{ Área } & \multirow[t]{2}{*}{ Error típ. ${ }^{a}$} & \multirow[t]{2}{*}{ Sig. asintóticab } & \multicolumn{2}{|c|}{ Intervalo de confianza asintótico al 95\% } \\
\hline & & & & Límite inferior & Límite superior \\
\hline \multicolumn{6}{|c|}{ Área bajo la curva España } \\
\hline Estado & .957 & .013 & .000 & .932 & .982 \\
\hline Rasgo & .923 & .019 & .000 & .886 & .960 \\
\hline \multicolumn{6}{|c|}{ Área bajo la curva Cuba } \\
\hline Estado & .885 & .019 & .000 & .848 & .922 \\
\hline Rasgo & .800 & .026 & .000 & .749 & .852 \\
\hline \multicolumn{6}{|c|}{ Área bajo la curva Colombia } \\
\hline Estado & .687 & .047 & .000 & .595 & .779 \\
\hline Rasgo & .536 & .052 & .436 & .435 & .638 \\
\hline \multicolumn{6}{|c|}{ Área bajo la curva México } \\
\hline Estado & .899 & .013 & .000 & .874 & .924 \\
\hline Rasgo & .892 & .013 & .000 & .867 & .918 \\
\hline \multicolumn{6}{|c|}{ Área bajo la curvaJóvenes } \\
\hline Estado & .859 & .020 & .000 & .820 & .899 \\
\hline Rasgo & .782 & .026 & .000 & .730 & .834 \\
\hline \multicolumn{6}{|c|}{ Área bajo la curva Adultos } \\
\hline Estado & .855 & .026 & .000 & .804 & .905 \\
\hline Rasgo & .836 & .026 & .000 & .785 & .887 \\
\hline \multicolumn{6}{|c|}{ Área bajo la curvaAncianos } \\
\hline Estado & .907 & .013 & .000 & .882 & .932 \\
\hline Rasgo & .820 & .018 & .000 & .784 & .856 \\
\hline \multicolumn{6}{|c|}{ área bajo la curvaHombres } \\
\hline Estado & .887 & .017 & .000 & .854 & .921 \\
\hline Rasgo & .819 & .021 & .000 &, 778 & .860 \\
\hline \multicolumn{6}{|c|}{ Área bajo la curvaMujeres } \\
\hline Estado & .875 & .013 & .000 & .850 & .900 \\
\hline Rasgo & .807 & .016 & .000 & .776 & .838 \\
\hline
\end{tabular}

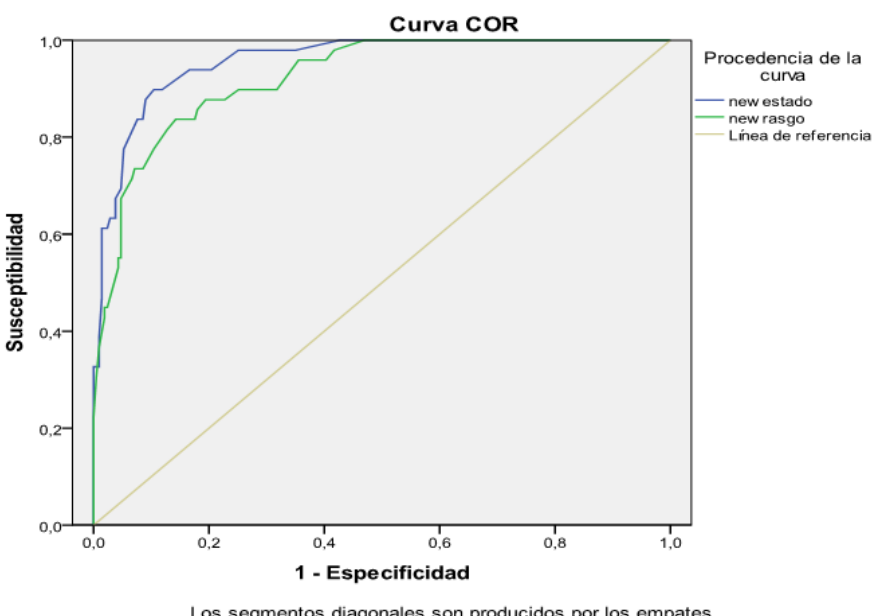

Los segmentos diagonales son producidos por los empates.

Figura 1. Curva COR España

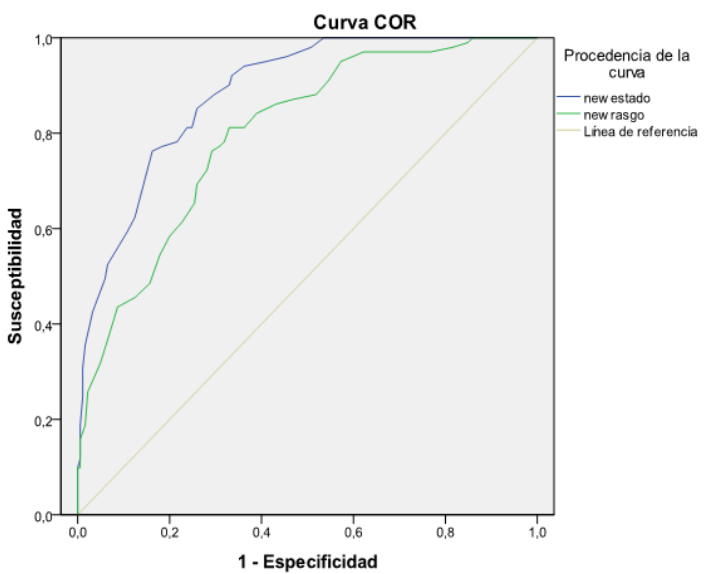

Los segmentos diagonales son producidos por los empates.

Figura 2. Curva COR Cuba 


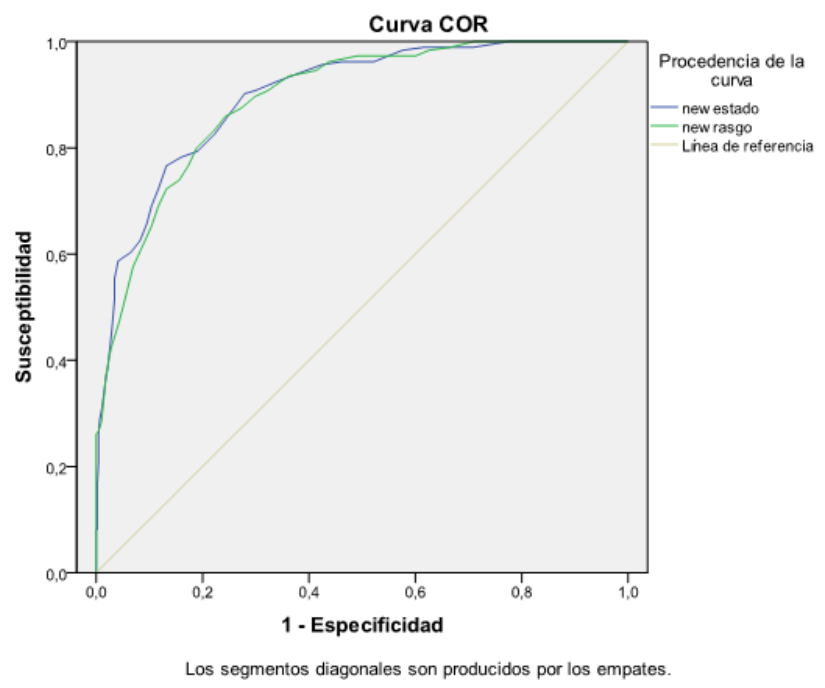

Figura 3. Curva COR México

Tabla 2. Valores de punto de corte recomendados para España, México y Cuba

\begin{tabular}{|c|c|c|c|c|c|}
\hline \multicolumn{6}{|l|}{ España } \\
\hline \multicolumn{3}{|c|}{ Estado } & \multicolumn{3}{|c|}{ Rasgo } \\
\hline $\begin{array}{l}\text { Positivo si es mayor o } \\
\text { igual que }\end{array}$ & Sensibilidad & 1 - Especificidad & $\begin{array}{l}\text { Positivo si es mayor o } \\
\text { igual que }\end{array}$ & Sensibilidad & 1 - Especificidad \\
\hline 36.50 & .961 & .250 & 43.50 & .882 & .288 \\
\hline 37.50 & .922 & .203 & 44.50 & .882 & .264 \\
\hline 38.50 & .922 & .165 & 45.50 & .882 & .250 \\
\hline 39.50 & .902 & .142 & 46.50 & .863 & .226 \\
\hline 40.50 & .882 & .118 & 47.50 & .863 & .193 \\
\hline 41.50 & .882 & .104 & 48.50 & .843 & .179 \\
\hline 42.50 & .863 & .090 & 49.50 & .824 & .175 \\
\hline 43.50 & .824 & .085 & 50.50 & .824 & .142 \\
\hline 44.50 & .824 & .075 & 51.50 & .804 & .127 \\
\hline \multirow[t]{5}{*}{45.50} & .745 & .052 & 52.50 & .765 & .104 \\
\hline & & & 53.50 & .725 & .085 \\
\hline & & & 54.50 & .725 & .071 \\
\hline & & & 55.50 & .706 & .066 \\
\hline & \multicolumn{5}{|c|}{ Cuba } \\
\hline 38.50 & .882 & .296 & 44.50 & .765 & .290 \\
\hline 39.50 & .853 & .258 & 45.50 & .725 & .280 \\
\hline 40.50 & .814 & .247 & & & \\
\hline 41.50 & .814 & .237 & & & \\
\hline 42.50 & .784 & .215 & & & \\
\hline 43.50 & .775 & .183 & & & \\
\hline 44.50 & .765 & .161 & & & \\
\hline \multicolumn{6}{|c|}{ México } \\
\hline 35.50 & .915 & .288 & 41.50 & .847 & .283 \\
\hline 36.50 & .881 & .258 & 42.50 & .847 & .253 \\
\hline 37.50 & .847 & .217 & 43.50 & .831 & .202 \\
\hline 38.50 & .831 & .192 & 44.50 & .797 & .182 \\
\hline \multirow[t]{2}{*}{39.50} & .746 & .167 & 45.50 & .780 & .162 \\
\hline & & & 46.50 & .729 & .146 \\
\hline
\end{tabular}

La estadística descriptiva por países, grupos de edad y sexo se presenta en la tabla 3 y los resultados del ANOVA multifactorial se muestran en la tabla 4 . Se encontraron diferencias significativas para ambas subescalas atribuibles al sexo, al país, la edad y a la interacción entre sexo y país, así como a la interacción entre país y edad. Correspondieron a las mujeres y los ancianos las mayores puntuaciones en ambas subescalas, en la escala rasgo las mayores puntuaciones las obtuvieron los colombianos, mientras que cubanos y mexicanos obtuvieron las mayores medias marginales estimadas para la escala estado. 
Tabla 3. Estadística descriptiva del IDERE por países, grupos de edad y sexo.

\begin{tabular}{|c|c|c|c|c|c|c|c|c|c|c|c|c|c|c|c|c|c|c|}
\hline & & & & $\overline{P a 1}$ & & & & & & & $\overline{\mathrm{Ed}}$ & des & & & & Sex & $\mathrm{SOS}$ & \\
\hline & Españ & & Cuba & & Colom & abia & Méxic & & Jóven & & Adultc & & Ancial & nos & Mujer & & Homb & \\
\hline & $\bar{E}$ & $\mathrm{R}$ & $E$ & $\mathrm{R}$ & $E$ & $\mathrm{R}$ & $E$ & $\mathrm{R}$ & $\mathrm{E}$ & $\mathrm{R}$ & $E$ & $\mathrm{R}$ & $E$ & $\mathrm{R}$ & $E$ & $\mathrm{R}$ & $E$ & $\mathrm{R}$ \\
\hline$\overline{\mathrm{M}}$ & 37.25 & 42.76 & 39.48 & 42.64 & 35.78 & 46.87 & 35.79 & 40.87 & 15.25 & 24.25 & 16.07 & 24.43 & 21.94 & 27.40 & 38.34 & 44.73 & 35.05 & 42.01 \\
\hline $\mathrm{ME}$ & 10.87 & 13.84 & 11.24 & 11.60 & 8.64 & 6.51 & 9.28 & 10.67 & 9.47 & 10.95 & 10.36 & 11.90 & 12.01 & 12.27 & 10.581 & 11.165 & 9.083 & 10.34 \\
\hline Min & 20 & 20 & 20 & 19 & 21 & 24 & 20 & 18 & 0 & 0 & 0 & 0 & 0 & 0 & 20 & 21 & 20 & 18 \\
\hline Máx & 76 & 81 & 76 & 78 & 66 & 65 & 70 & 75 & 52 & 63 & 56 & 63 & 56 & 67 & 76 & 81 & 76 & 72 \\
\hline
\end{tabular}

Leyenda: E: Subescala estado del IDERE, R: Subescala rasgo del IDERE, Min: valor mínimo , Máx: valor máximo,

Tabla 4. Resultados del ANOVA multifactorial (pruebas de los efectos inter-sujetos)

\begin{tabular}{|c|c|c|c|c|c|c|c|c|c|c|}
\hline Origen & $\begin{array}{l}\text { Variable } \\
\text { dependiente }\end{array}$ & & $\begin{array}{l}\text { Suma de } \\
\text { cuadrados } \\
\text { tipo III }\end{array}$ & gl & $\begin{array}{l}\text { Media } \\
\text { cuadrática }\end{array}$ & $\mathrm{F}$ & $p$ & $\begin{array}{l}\text { Eta }^{2} \\
\text { parcial }\end{array}$ & $\begin{array}{l}\text { Parámetro de no } \\
\text { centralidad } \\
\text { Parámetro }\end{array}$ & $\begin{array}{l}\text { Potencia } \\
\text { observada }^{\mathrm{b}}\end{array}$ \\
\hline \multirow{2}{*}{$\begin{array}{l}\text { Modelo } \\
\text { corregido }\end{array}$} & \multirow[t]{2}{*}{ dimension1 } & estado & $32425.482^{a}$ & 23 & 1409.804 & 12.944 & .000 & .171 & 297.721 & 1.000 \\
\hline & & rasgo & $21837.085^{c}$ & 23 & 949.438 & 7.479 & .000 & .107 & 172.009 & 1.000 \\
\hline \multirow[t]{2}{*}{ Intersección } & \multirow[t]{2}{*}{ dimension1 } & estado & 316019.257 & 1 & 316019.257 & 2901.592 & .000 & .668 & 2901.592 & 1.000 \\
\hline & & rasgo & 690677.563 & 1 & 690677.563 & 5440.412 & .000 & .790 & 5440.412 & 1.000 \\
\hline \multirow[t]{2}{*}{ Sexo } & \multirow[t]{2}{*}{ dimension1 } & estado & 4499.531 & 1 & 4499.531 & 41.313 & .000 & .028 & 41.313 & 1.000 \\
\hline & & rasgo & 4024.226 & 1 & 4024.226 & 31.699 & .000 & .021 & 31.699 & 1.000 \\
\hline \multirow[t]{2}{*}{ País } & \multirow[t]{2}{*}{ dimension1 } & estado & 1590.813 & 3 & 530.271 & 4.869 & .002 & .010 & 14.606 & .908 \\
\hline & & rasgo & 6721.268 & 3 & 2240.423 & 17.648 & .000 & .035 & 52.943 & 1.000 \\
\hline \multirow[t]{2}{*}{ Edad } & \multirow[t]{2}{*}{ dimension1 } & estado & 8305.956 & 2 & 4152.978 & 38.131 & .000 & .050 & 76.263 & 1.000 \\
\hline & & rasgo & 1497.158 & 2 & 748.579 & 5.896 & .003 & .008 & 11.793 & .877 \\
\hline \multirow[t]{2}{*}{ Sexo * País } & \multirow[t]{2}{*}{ dimension1 } & estado & 1210.463 & 3 & 403.488 & 3.705 & .011 & .008 & 11.114 & .807 \\
\hline & & rasgo & 1780.134 & 3 & 593.378 & 4.674 & .003 & .010 & 14.022 & .896 \\
\hline \multirow[t]{2}{*}{ Sexo $*$ edad } & \multirow[t]{2}{*}{ dimension1 } & estado & 264.638 & 2 & 132.319 & 1.215 & .297 & .002 & 2.430 & .266 \\
\hline & & rasgo & 33.344 & 2 & 16.672 & .131 & .877 & .000 & .263 & .070 \\
\hline \multirow[t]{2}{*}{ País * edad } & \multirow[t]{2}{*}{ dimension1 } & estado & 6848.723 & 6 & 1141.454 & 10.480 & .000 & .042 & 62.883 & 1.000 \\
\hline & & rasgo & 5901.998 & 6 & 983.666 & 7.748 & .000 & .031 & 46.490 & 1.000 \\
\hline \multirow{2}{*}{\multicolumn{2}{|c|}{$\begin{array}{l}\text { Sexo * País dimension1 } \\
* \text { edad }\end{array}$}} & estado & 812.717 & 6 & 135.453 & 1.244 & .281 & .005 & 7.462 & .495 \\
\hline & & rasgo & 549.945 & 6 & 91.657 & .722 & .632 & .003 & 4.332 & .290 \\
\hline \multirow[t]{2}{*}{ Error } & \multirow[t]{2}{*}{ dimension1 } & estado & 157160.524 & 1443 & 108.912 & & & & & \\
\hline & & rasgo & 183193.418 & 1443 & 126.953 & & & & & \\
\hline \multirow[t]{2}{*}{ Total } & \multirow[t]{2}{*}{ dimension1 } & estado & 672085.000 & 1467 & & & & & & \\
\hline & & rasgo & 1170072.000 & 1467 & & & & & & \\
\hline \multirow{2}{*}{\multicolumn{2}{|c|}{$\begin{array}{l}\text { Total corre- dimension1 } \\
\text { gida }\end{array}$}} & estado & 189586.005 & 1466 & & & & & & \\
\hline & & rasgo & 205030.503 & 1466 & & & & & & \\
\hline
\end{tabular}

a. $\mathrm{R}$ cuadrado $=.171$ ( $\mathrm{R}$ cuadrado corregida $=.158)$

b. Calculado con alfa $=, 05$

c. $\mathrm{R}$ cuadrado $=.107(\mathrm{R}$ cuadrado corregida $=.092)$

Como era de esperar ambas subescalas tienen correlaciones significativas y positivas (tabla 5) entre ellas y con el CES-D.

Tabla 5. Correlaciones entre IDERE y CES-D.

\begin{tabular}{lllll}
\hline & & $\begin{array}{l}\text { IDERE } \\
\text { estado }\end{array}$ & $\begin{array}{l}\text { IDERE } \\
\text { rasgo }\end{array}$ & CES-D \\
\hline IDERE- & Correlación de Pearson & 1 & $.770\left(^{* *}\right)$ & $.683\left(^{* *}\right)$ \\
estado & Sig. (bilateral) & 1675 & .000 & .000 \\
& $\mathrm{~N}$ & 1570 & 1337 \\
IDERE & Correlación de Pearson & $.770\left(^{* *}\right)$ & 1 & $.556\left(^{* *}\right)$ \\
rasgo & Sig. (bilateral) & .000 & & .000 \\
& $\mathrm{~N}$ & 1570 & 1600 & 1270 \\
CES-D & Correlación de Pearson & $.683(* *)$ & $.556\left(^{* *}\right)$ & 1 \\
& Sig. (bilateral) & .000 & .000 & \\
& $\mathrm{~N}$ & 1337 & 1270 & 1611 \\
\hline
\end{tabular}

** La correlación es significativa al nivel .01 (bilateral).

\section{Discusión y Conclusiones}

Debe recordarse que el propósito original del IDERE es identificar y cuantificar la intensidad de un estado emocional de matiz depresivo (escala estado) así como diagnosticar el grado de vulnerabilidad personal a sufrir depresión (escala rasgo). Téngase en cuenta que la consigna de la escala estado se refiere a lo que siente la persona en el momento en que está respondiendo el cuestionario; y la de la escala estado a la depresión "habitual"; y los manuales actuales de clasificación son bastante restrictivos acerca del tiempo de duración de los síntomas necesario para establecer el diagnóstico (al menos dos semanas para el diagnóstico de episodio depresivo mayor y al menos dos años para el diagnóstico de distimia, por ejemplo) por lo que no debe usarse como instrumento de primera línea para clasificar a los pacientes en determinadas categorías, lo que por otra parte, podría afir- 
marse también de muchos otros instrumentos para el diagnóstico de la depresión.

Dicho lo anterior, los resultados de esta investigación apuntan a que debe considerarse que, al menos para Cuba, España y México, tanto las personas que admiten sentimientos y síntomas depresivos en el momento de la evaluación (depresión estado) como las personas que tienen habitualmente autoevaluaciones negativas acerca de sí misma y del mundo (depresión rasgo) pueden estar presentando un trastorno depresivo, aunque el IDERE no permita precisar cuál.

El IDERE valora más los componentes cognitivos de la depresión, fundamentalmente los relacionados con el autoconcepto (sobre todo en la escala rasgo) y, la autoevaluación que hace la persona acerca de la presencia de ciertos síntomas de depresión y malestar (sobre todo en la escala estado), por tanto, era de esperar que la escala estado mostrara mayor sensibilidad para detectar a personas con depresión clínica que la escala rasgo, tal y como se encontró en este estudio, de manera consistente en los diferentes países, en ambos sexos y en los tres grupos de edades.

Resulta de interés que para la muestra colombiana no se obtuvieron indicadores satisfactorios, por lo que el IDERE deberá utilizarse con cautela hasta que no se precisen datos para este país.

En el presente trabajo se obtienen diferentes puntos de corte para Cuba, México y España que pueden usarse teniendo en cuenta que un proceder diagnóstico no tiene un solo valor de sensibilidad y especificidad, por tanto los investigadores o clínicos interesados en identificar posibles casos de depresión deberán seleccionar el que mejor convenga a sus propósitos. Estos puntos de corte propuestos deben considerarse como provisionales ya que no se ha trabajado con muestras representativas.

Serán necesarios futuros estudios para precisar la utilidad del IDERE en el diagnóstico clínico de los diferentes trastornos depresivos pues en esta investigación el procedimiento utilizado para detectar a personas con posible depresión se basó en las respuestas de los sujetos a diversos cuestiona-

\section{Referencias}

Agudelo, D. (2009). Propiedades psicométricas del Inventario de Depresión Estado/Rasgo (IDER) con adolescentes y universitarios de la ciudad de Bucaramanga. Pensamiento Psicológico, 5 (12),139-160.

Agudelo, D.M., Carretero-Dios, H., Blanco, A., Pitti, C., Spielberger, C.D. y Buela-Casal, G. (2005). Evaluación del componente afectivo de la depresión: análisis factorial del ST/DEP revisado Salud Mental, 28(3),33-41.

Agudelo, D., Spielberger, C.D., Buela-Casal, G. (2007): La depresión, ¿un trastorno dimensional o categorial? Salud Mental, 30(3),20-28.

American Psychiatric Association (2000). Manual Diagnóstico y Estadístico de los Trastornos Mentales (4th ed., Revisited Text). Barcelona: Mason.

Beekman, A., Deeg, D., van Tilburg, T., Smit, J., Hooijer, C., van Tilburg, W. (1995). Major and minor depression in later life: a study of prevalence and risk factors. Journal of Affectives Disorders, 36, 65-75.

Cabañero-Martínez, M.C., Cabrero-García, J., Richart-Martínez, M., MuñozMendoza, C.L.y Reig-Ferrer, A. (2007). Revisión estructurada de las escalas de depresión en personas mayores. International Journal of Clinical and Health Psychology, 7 (3),823-846. rios, lo que no permite obtener un diagnóstico verdadero, sino solamente presuntivo.

También será necesario determinar si la alta y significativa correlación entre las escalas rasgo y estado podría ser expresión no sólo de los intervínculos genéticos entre ambas formas de manifestarse la depresión como se plantea en el enfoque personal, sino también de la comorbilidad de trastornos como la distimia y la depresión mayor, que algunos autores han descrito con el término "doble depresión" (Keller y Shapiro, 1982).

En la presente investigación se encontraron diferencias en las puntuaciones del IDERE atribuibles al género, país y edad; pero estos resultados se deben interpretar con cautela ya que una limitación de esta investigación es que las muestras fueron seleccionadas por conveniencia y por ende, las diferencias encontradas podrían explicarse por especificidades de las muestras debidas a las oportunidades para acceder a las poblaciones que tuvieron los investigadores. Esta puede ser una explicación razonable, sobre todo, de las divergencias encontradas entre la muestra de Colombia y las de los restantes países.

A pesar de las limitaciones señaladas a la presente investigación, es conveniente recordar que el desempeño clínico de una prueba puede ser medido en términos de precisión diag-nóstica o sea, de su habilidad para clasificar correctamente a los sujetos en subgrupos clínicamente relevantes. En este sentido, la presente investigación muestra que ambas subescalas del IDERE tienen una buena precisión para diagnosticar depresión clínica según los criterios del DSM-IV-R en personas de ambos sexos, de diferentes grupos de edades, de 3 países distintos (Cuba, México y España). El resultado de este análisis, conjuntamente con el hecho de que se encontraron correlaciones significativas con el CES-D, un instrumento validado y ampliamente usado para el pesquisaje de depresión clínica, habla a favor de la validez de criterio y convergente del IDERE y de su valor para la identificación de personas con posible depresión en países iberoamericanos.

Campos, A., Díaz, L., Rueda, G., Cadena, L., Hernández, N. (2007). Psychometric properties of CES-D Scale among colombian adults from the general population. Revista Colombiana de Psiquiatría,36(4), 664-74.

Consejo de Organizaciones Internacionales de Ciencias Médicas y Organización Mundial de la Salud (1982). Proposed International Guidel-lines for Biomedical research involving human subjects. Ginebra: OMS (document)

Chacón, M., Grau, J.A., Martín, M. (1994). Estados emocionales generados por situaciones laborales estresantes en enfermeros oncólogos. Reporte final de investigación no publicado, Instituto Nacional de Oncología y Radiobiología, La Habana, Cuba.

Díaz, D., Blanco, A., Horcajo, J. y Valle, C. (2007). La aplicación del modelo de estado completo de salud al estudio de la depresión. Psicothema, 19(2), 286294.

Dowd, E.T. (2004). Depression: Theory, assessment, and new directions in practice. International Journal of Clinical and Health Psychology, 4, 413-423.

Enríquez, J.A., Martín, M., Riquelme, A. (2008). Evaluación de la Escala del Centro de Estudios Epidemiológicos de la Depresión (CES-D). Revista del Hospital Psiquiátrico de La Habana, 5(3), 15-22. 
González, M., Martín, M., Grau, J.A. y Lorenzo, A. (2007). Instrumentos de evaluación psicológica para el estudio de la ansiedad y la depresión. En M. González (Ed), Instrumentos de Evaluación Psicológica. (cap.10, pp. 164223) La Habana: Editorial Ciencias Médicas.

Grau, J., Martín, M., Portero, D. (1991). El sistema CUBANSIOPAT para el diagnóstico de las formas patológicas y no patológicas de ansiedad. Ponencia presentada en el XXIII Congreso Interamericano de Psicología, San José, Costa Rica.

Grau, J., Martín, M., Portero, D. (1993). Estrés, ansiedad, personalidad: resultados de las investigaciones cubanas efectuadas sobre la base del enfoque personal. Revista Interamericana de Psicología,27(1), 37-58.

Guarnaccia, P.J., Angel, R., Worobey, J.L. (1989). The factor structure of the CES-D in the Hispanic Health and Nutrition Examination Survey: The influences of ethnicity, gender, and language. Social Science and Medicine, 29,85-94.

Hybels, C., Blazer, D., Pieper, C. (2001). Toward a threshold for subthreshold depression: an analysis of correlates of depression by severity of symptoms using data from an elderly community sample. Gerontologist, 41,357-365.

Jiménez, P.J. (1993). Cuidados Paliativos al paciente oncológico terminal. Tesis para la obtención del título de Especialista de Primer Grado en Oncología, Instituto Nacional de Oncología y Radiobiología. La Habana, Cuba (no publicada).

Keller, M.B. y Shapiro, R.W. (1982). "Double Depression": superimposition of acute depressive episodes on chronic depressive disorders. American Journal of Psychiatry, 139,438-442.

Lechuga, M. (2007). Informe de resultados de los programas psicoeducativos desarrollados en cinco liceos de la Corporación de Capacitación y Empleo SOSOFA. Santiago de Chile, (manuscrito no publicado).

Martín, M. (2003) Inventario de Depresión Rasgo-Estado (IDERE). Manual de aplicación. Santiago de Chile: Acquaris.

Martín, M. y Grau, J.A. (1991). Evaluación de la ansiedad y la depresión en enfermos crónicos. Ponencia presentada en el XXIII Congreso Interamericano, San José de Costa Rica.

Martín, M. y Grau, J.A. (1993). La Psicología en los programas de atención a los enfermos crónicos. Ponencia presentada en el II Congreso Colombiano de Psicología de la Salud.

Martín, M., Grau, J.A., Grau, R. (2003). El Inventario de Depresión RasgoEstado (IDERE). Desarrollo de una versión cubana. Revista Terapias Psicológicas, 3(3), 23-31.

Martín, M., Grau, J.A., Ramírez, V.y Grau, R. (2001). El Inventario de Depresión Rasgo-Estado (IDERE): Desarrollo y Potencialidades. Revista Psicología.com5(1), http://www.psiquiatria.com/psicologia/revista/134/.

Martín, M., Grau, J.A., Ramírez, V. y Grau, R. (2005). El Inventario de Depresión rasgo-Estado. En P. Vera y L. Oblitas (Eds.) Manual de escalas y cuestionarios iberoamericanos en psicología clínica y de la salud. Bogotá: Psicom Editores (e-book).

Martín, M. y Lechuga, M. (2003). Factores psicosociales en el dolor agudo. En Derderian T (Ed) Guías Prácticas para el manejo del dolor agudo (pp.1525). Santiago de Chile: Merck.

Martín, M. y Lechuga, M. (2004). Factores psicosociales en el dolor en ancianos En Derderian T (Ed) Guías Prácticas para el manejo del dolor en ancianos (pp.12-20). Santiago de Chile: Merck.

Mellor,B. y Aragona,M. (2009).De la categoría a la dimensión: una mirada crítica a la evolución de la nosografía psiquiátrica Revista de la Asociación. Española de Neuropsiquiatría, 39(103), 217-228.

Navarro, J.J., Marchena, E., González, D., Manjón, M., Pallarés, M.J. y Martín, M. (1996). Aspectos Psicológicos del Dolor. En LM Torres (ED) Medicina del Dolor. (pp.333-456) Madrid: Masson.
Ocampo, L.E. (2007). Análisis Correlacional del Cuestionario de Depresión Estado/Rasgo con una muestra de adolescentes y universitarios de la ciudad de Medellín (Colombia).Revista Psicología desde el Caribe, Universidad del Norte. 20, 28-49.

Pérez, R., Riquelme, A., Martín, M., Enriquez, J.A. y Ortigosa, J.M. (2009). Cuestionario de síntomas depresivos. Ponencia presentada en el XIV Congreso Internacional de la Asociación Médica del Caribe AMECA CMA/CTC, La Habana, Centro de Investigaciones Médico-Quirúrgicas.

Radolff, J.S. (1977). The CES-D scale: a self-report depression scale for research in the general population. Applied Psychological Measurment, 1,385401.

Ramírez, V., Grau, J., Martín, M. y Grau, R. (1986). Construcción de un inventario para evaluar la depresión como rasgo y como estado. Reporte de investigación no publicado, Facultad de Psicología de la Universidad Central de las Villas, Cuba.

Riquelme, A., Buendía, J. y López, A.I. (2006). Desarrollo y validación de un instrumento para la evaluación de la depresión en ancianos. Psicothema, 18(2), 288-292.

Riquelme, M., Martín, M., Ortigosa, J. y Lechuga M. (2008).Depresión y envejecimiento: algunas cuestiones de interés respecto a la investigación epidemiológica. Revista Multidisciplinar de Gerontología, 18(1), 76-80 http://dialnet.unirioja.es/servlet/listaarticulos?tipo_busqueda=VOLUME N\&revista_busqueda $=1996 \&$ clave_busqueda $=18$.

Salgado, N. y Maldonado, M. (1994). Características psicométricas de la escala de depresión del Centro de Estudios Epidemiológicos de la Depresión en mujeres mexicanas adultas de áreas rurales. Revista de Salud Pública de México, 36, 200-209.

Soler, J., Pérez-Sola, V., Puigdemont, D., Pérez-Blanco, J., Figueres, M., Alvarez, E. (1998). Estudio de validación del Center for Epidemiologic StudiesDepresion (CES-D) en una población española de pacientes con trastornos afectivos. Actas Luso-Españolas de Neurología y Psiquiatría, 25, 243-249.

Spielberger, C.D., Agudelo, D., Carretero-Dios, H., De Los Santos-Roig, M. y Buela-Casal, G. (2004). Análisis de reactivos de la Versión Experimental castellana del cuestionario para la depresión Estado-Rasgo (ST-DEP). Análisis y Modificación de Conducta, 30, 139-160.

Spielberger, C.D., Carretero-Dios, H., De Los Santos-Roig, M. y Buela-Casal, G. (2002 a). Spanish experimental version of the state-trait depression questionnaire (ST-DEP): State sub-scale (S-DEP). Revista Internacional de Psicología Clínica y de la Salud, 2, 71-89.

Spielberger, C.D., Carretero-Dios, H., De Los Santos-Roig, M. y Buela-Casal, G. (2002b). Spanish experimental version of the state-trait depression questionnaire (ST-DEP): Trait sub-scale (T-DEP). Revista Internacional de Psicología Clínica y de la Salud, 2, 51-69.

Spielberger, C.D., Ritterband, L.M., Reheiser, E.C. y Brunner, T.M., (2003). The nature and measurement of depression. International Journal of Clinical and Health Psychology, 3(2), 209-234.

Vázquez, F.L., Blanco, V. y López, M. (2007). An adaptation of the Center for Epidemiologic Studies Depression Scale for use in non-psychiatric Spanish populations. Psychiatry Research, 149, 247-252.

Zunzunegui, M.V., Delgado, M., Pérez, E., Yagüe, A., Illescas, M.L. y León, V. (1998). Validación de la escala CES_D para la medida de la sintomatología depresiva en una población de personas mayores española. Revista Multidisciplinar de Gerontología, 8,156-161.

(Articulo recibido: 23-1-2012, revisado: 18-1-2012, aceptado: 20-2-2012 